\title{
The Prognostic Value of Irradiated Lung Volumes on the Prediction of Intra-/ Post-Operative Mortality in Patients after Neoadjuvant Radiochemotherapy for Esophageal Cancer. A Retrospective Multicenter Study.
}

\author{
Philipp Günther Kup1 ${ }^{1}$ Carsten Nieder², Hans Geinitz ${ }^{3,4}$, Christoph Henkenberens 5 , Angela Besserer6, \\ Markus Oechsner ${ }^{4}$, Sabine Schill', Ralph Mücke',7, Vera Scherer ${ }^{4}$, Stephanie E. Combs ${ }^{4}$, Irenäus A. \\ Adamietz $^{1,8}$, Khashayar Fakhrian ${ }^{1,4,8}$ \\ 1. Department of Radiation Oncology, Marien Hospital Herne, Clinic of Ruhr-Universität Bochum, Herne, Germany. \\ 2. Department of Oncology and Palliative Medicine, Nordland Hospital Bodø, Norway. \\ 3. Department of Radiation Oncology, Krankenhaus der Barmherzigen Schwestern Linz, Linz, Austria. \\ 4. Department of Radiation Oncology, Klinikum rechts der Isar, Technische Universität München, Munich, Germany \\ 5. Department of Radiation Oncology, Medizinische Hochschule Hannover, Hannover, Germany. \\ 6. Department of Radiation Oncology, Charité Universitätsmedizin Berlin, Campus Benjamin Franklin, Berlin, Germany. \\ 7. Department of Radiation Oncology, Lippe Hospital, Lemgo, Germany. \\ 8. Department of Radiation Oncology, Sankt Josef Hospital Bochum, Clinic of Ruhr-University Bochum, Bochum, Germany.
}

$\square$ Corresponding author: Dr. Khashayar Fakhrian, Department of Radiation Oncology, Marienhospital Herne, Ruhr-University Bochum, Hoelkeskampring 40, 44625 Herne, Germany, Telephone: (+)49 (0)2323 499-1531, Fax: (+)49 (0)2323 499-306, Email: khfmed@yahoo.com

๑) 2015 Ivyspring International Publisher. Reproduction is permitted for personal, noncommercial use, provided that the article is in whole, unmodified, and properly cited. See http://ivyspring.com/terms for terms and conditions.

Received: 2014.10.13; Accepted: 2014.12.06; Published: 2015.01.20

\begin{abstract}
Purpose: To assess the association between dosimetric factors of the lung and incidence of intraand postoperative mortality among esophageal cancer $(E C)$ patients treated with neoadjuvant radiochemotherapy (N-RCT) followed by surgery $(\mathrm{S})$.

Methods and Materials: Inclusion criteria were: age $<85$ years, no distant metastases at the time of diagnosis, no induction chemotherapy, conformal radiotherapy, total dose $\leq 50.4 \mathrm{~Gy}$, and available dose volume histogram (DVH) data. One-hundred thirty-five patients met our inclusion criteria. Median age was 62 years. N-RCT consisted of 36 - 50.4 Gy (median 45 Gy), 1.8 - 2 Gy per fraction. Concomitant chemotherapy consisted of 5-Fluoruracil (5-FU) and cisplatin in 113 patients and cisplatin and taxan-derivates in 15 patients. Seven patients received a single cytotoxic agent. In 130 patients an abdominothoracal and in 5 patients a transhiatal resection was performed. The following dosimetric parameters were generated from the total lung DVH: mean dose, V5, V10, $\mathrm{V} 15, \mathrm{~V} 20, \mathrm{~V} 30, \mathrm{~V} 40, \mathrm{~V} 45$ and V50. The primary endpoint was the rate of intra- and postoperative mortality (from the start of N-RCT to 60 days after surgical resection).

Results: A total of ten postoperative deaths (7\%) were observed: 3 within 30 days (2\%) and 7 between 30 and 60 days after surgical intervention (5\%); no patient died during the operation. In the univariate analysis, weight loss ( $\geq 10 \%$ in 6 months prior to diagnosis, risk ratio: $1.60,95 \% \mathrm{Cl}$ : 0.856-2.992, $p=0.043$ ), Eastern Cooperative Oncology Group-performance status (ECOG 2 vs. 1, risk ratio: $1.931,95 \% \mathrm{Cl}: 0.898-4.150, \mathrm{p}=0.018$ ) and postoperative pulmonary plus non-pulmonary complications (risk ratio: $2.533,95 \% \mathrm{Cl}: 0.978-6.563, \mathrm{p}=0.004$ ) were significantly associated with postoperative mortality. There was no significant association between postoperative mortality and irradiated lung volumes. Lung V45 was the only variable which was significantly associated with higher incidence of postoperative pulmonary plus non-pulmonary complications $(\operatorname{Exp}(B): 1.285$,
\end{abstract}


$95 \% \mathrm{Cl} 1.029-1.606, \mathrm{p}=0.027)$, but not with the postoperative pulmonary complications $(\operatorname{Exp}(\mathrm{B})$ : $1.249,95 \% \mathrm{Cl} 0.999-1.561, \mathrm{p}=0.051)$.

Conclusions: Irradiated lung volumes did not show relevant associations with intra- and postoperative mortality of patients treated with moderate dose (36-50.4 Gy) conventionally fractionated conformal radiotherapy combined with widely used radiosensitizers. Postoperative mortality was significantly associated with greater weight loss, poor performance status and development of postoperative complications, but not with treatment-related factors. Limiting the volume of lung receiving higher radiation doses appears prudent because of the observed association with risk of postoperative complications.

Key words: Esophageal cancer, Neoadjuvant radiochemotherapy, Dose-volume histogram, Lung toxicity, Postoperative mortality

\section{Introduction}

Tri-modality treatment (TMT), defined as radiochemotherapy followed by surgical resection, is the standard of care for patients with locally advanced squamous cell esophageal cancer (ESCC) who can tolerate aggressive therapy (1-4). Although the English MAGIC trial of perioperative chemotherapy without radiation had several shortcomings considering design, protocol, selection of patients, randomization/ homogeneity of patient groups, quality of surgical care and statistical evaluation, it changed very soon the standard of care for patients with distal esophageal adenocarcinoma (EAC), at least in Europe (5). Recently, the landmark study CROSS revitalized the discussion around efficacy of TMT in the management of EAC, which are classified as adenocarcinoma of esophagogastric junction (AEG), AEG I and AEG II by Siewert et al (6). Patients with AEG III are usually classified and treated like gastric cancer. Since publication of the randomized CROSS study, we know that carefully tailored TMT does not increase the postoperative mortality compared to surgery alone (1). In that study, patients randomized to TMT were treated with radiotherapy $(41.4 \mathrm{~Gy})$ and concomitant chemotherapy with weekly carboplatin (Area under the curve of 2 (AUC2), on days 1, 8, 15, 22, and 29) and paclitaxel at a dose of $50 \mathrm{mg} / \mathrm{m}^{2}$. A pathological complete response was achieved in 47 of 161 patients $(29 \%)$ who underwent resection after neoadjuvant radiochemotherapy (N-RCT). Postoperative complications were similar in the two treatment arms, and in-hospital mortality was $4 \%$ in both groups. Median overall survival was 49.4 months in the TMT group versus 24.0 months in the surgery alone group $(p=0.003)$.

The major concern against TMT in the management of esophageal cancer is a possible increase in intra- or postoperative mortality, largely due to irradiation of the lungs and consecutive pulmonary complications (7-9). Several dose-volume modeling studies were performed, which reported a correlation between adverse events and mean lung dose, lung volume irradiated with 5 Gy (V5), lung V10, lung V15, lung V20 or lung V30 (7,10-12). Of note, in these studies a considerable proportion of patients was treated with induction chemotherapy (7), pulmotoxic cytotoxic agents (8), unusual (very large) radiation fields (9) or higher radiation dose per fraction (13). These factors might have influenced the risk of toxicity and complications. An older meta-analysis published in 2004 (14) showed that postoperative mortality was higher in patients treated with TMT compared to those treated with surgery alone. However, this conclusion was mainly derived from the increased mortality observed in the largest randomized trial in the analysis, in which a dose per fraction of $3.7 \mathrm{~Gy}$ was used. More data from patients treated with standard fractionation to limited target volumes are needed to shed more light on the safety of TMT, the risk of pulmonary toxicity, and factors predicting pulmonary toxicity. The main aim of our study was to assess the association between dosimetric factors of the lung and rate of intra- and postoperative mortality among patients treated with TMT.

\section{Methods and Materials}

Data from 551 patients from 6 centers (Table 1) were retrospectively reviewed to screen for eligible patients. Inclusion criteria for our study were: age $<85$ years, no distant metastases at the time of diagnosis, no induction chemotherapy, three-dimensional conformal radiotherapy, available dose-volume histogram (DVH) data, radiotherapy dose $\leq 50.4$ Gy and complete TMT including surgical resection. One-hundred thirty-five patients met these inclusion criteria. The characteristics of these 135 patients are displayed in Table 2. Median age was 62 years (range: 33-80 years). N-RCT consisted of 36-50.4 Gy (median 45 Gy) (1.8-2 Gy per fraction). Concomitant chemotherapy consisted of 5-FU and platin-derivates in 113 patients, and cisplatin and taxan-derivates in 15 pa- 
tients. Seven patients received a single cytotoxic agent. In 130 patients an abdominothoracal and in 5 patients a hiatal resection was performed. The interval between end of N-RCT and surgery was 4-6 weeks. The following dosimetric parameters were generated from the DVH for total lung (left plus right lung): mean lung dose, V5, V10, V15, V20, V30, V40, V45 and V50. The primary study endpoint was treatment-related mortality (from start of N-RCT to 60 days after surgical resection). Mortality was defined as death from any cause. Serious postoperative complications were defined as pneumonia, acute respiratory distress syndrome (ARDS), heart attack, sepsis and anastomosis insufficiency, that developed postoperatively within 60 days after surgery or before discharge. Pulmonary complications were defined as ARDS and pneumonia. The rate of serious complications was evaluated as a secondary endpoint. Toxicities were retrospectively assessed according to the National Cancer Institute Common Toxicity Criteria (NCI-CTC) Version 3.0. Postoperative complications were defined as pneumonia, acute respiratory distress syndrome (ARDS), heart attack, sepsis and anastomosis insufficiency, which developed postoperatively within 60 days after surgery or before discharge. Pulmonary complications were defined as ARDS and pneumonia, which developed postoperatively within 60 days after surgery or before discharge.

Table 1: Participating centers

\begin{tabular}{ll}
\hline Center & No. of patients \\
\hline RdI, TUM, Munich, Germany & 58 \\
BHS, Linz, Austria & 35 \\
MHH, Hannover, Germany & 21 \\
RUB, Bochum, Germany & 12 \\
CBF, Berlin, Germany & 9 \\
\hline RdI: Klinikum rechts der Isar & \\
TUM: Technische Universität München & \\
BHS: Krankenhaus der Barmherzigen Schwestern, Linz \\
MHH: Medizinische Hochschule Hannover \\
RUB: Ruhr-University Bochum \\
CBF: Campus Benjamin Franklin, Charité Berlin
\end{tabular}

Table 2: Patients' characteristics and association with incidence of postoperative complications or death

\begin{tabular}{|c|c|c|c|c|c|}
\hline Characteristics & $\mathbf{N}(\%)$ & Complications (\%) & p-value & POD $\pm(\%)$ & p-value \\
\hline Age, median (range) & 62 y $(33-80 y)$ & & 0.204 & & 0.131 \\
\hline Gender & & & 0.379 & & 1.000 \\
\hline Male & $102(75)$ & $26(26)$ & & $8(8)$ & \\
\hline Female & $33(25)$ & $11(33)$ & & $2(6)$ & \\
\hline Alcohol abuse history & & & 1.000 & & 0.146 \\
\hline Yes & $78(58)$ & $15(28)$ & & $6(8)$ & \\
\hline No & $55(41)$ & $22(28)$ & & $3(6)$ & \\
\hline Unknown & $2(1)$ & $0(0)$ & & $1(50)$ & \\
\hline Smoking history & & & 0.703 & & 0.326 \\
\hline Yes & $66(49)$ & $17(26)$ & & $3(5)$ & \\
\hline No & $69(51)$ & $20(29)$ & & $7(10)$ & \\
\hline Co-morbidities $¥$ & & & 0.336 & & 0.328 \\
\hline Yes & $70(52)$ & $22(31)$ & & $7(10)$ & \\
\hline No & $65(48)$ & $15(23)$ & & $3(5)$ & \\
\hline Weight loss $\geq 10 \% 0^{* * *}$ & & & 0.166 & & 0.043 \\
\hline Yes & $30(22)$ & $5(17)$ & & $5(17)$ & \\
\hline No & $105(78)$ & $32(31)$ & & $5(5)$ & \\
\hline ECOG-PS & & & $0.832^{* *}$ & & $0.018^{*}$ \\
\hline $0-1$ & 99 (73) & $26(26)$ & & $4(4)$ & \\
\hline 2 & $34(26)$ & $10(29)$ & & $6(18)$ & \\
\hline Unknown & $2(1)$ & & & & \\
\hline Histology & & & 0.443 & & 0.637 \\
\hline Squamous cell & $118(87)$ & $35(30)$ & & $10(9)$ & \\
\hline Adenocarcinoma & $16(12)$ & $2(12)$ & & $0(0)$ & \\
\hline Undifferentiated & $1(1)$ & & & & \\
\hline cT Stage & & & 0.375 & & 0.337 \\
\hline T1-2 & $16(12)$ & $6(37)$ & & $2(12)$ & \\
\hline T3-4 & $119(88)$ & $31(26)$ & & $8(7)$ & \\
\hline cN Stage & & & 0.789 & & 1.000 \\
\hline 0 & $20(15)$ & $6(30)$ & & $1(5)$ & \\
\hline 1 & $115(85)$ & $31(27)$ & & $9(8)$ & \\
\hline Localisation & & & 0.550 & & 0.916 \\
\hline Suprabifurcational & $26(19)$ & $10(39)$ & & $2(8)$ & \\
\hline Infrabifurcational & $70(52)$ & $17(25)$ & & $6(9)$ & \\
\hline Overlapping & $38(28)$ & $10(26)$ & & $2(5)$ & \\
\hline Unknown & $1(1)$ & $0(0)$ & & $0(0)$ & \\
\hline Length & & & 1.000 & & 1.000 \\
\hline
\end{tabular}




\begin{tabular}{|c|c|c|c|c|c|}
\hline$<10 \mathrm{~cm}$ & $130(96)$ & $36(28)$ & & $10(8)$ & \\
\hline$\geq 10 \mathrm{~cm}$ & $5(4)$ & $1(20)$ & & $0(0)$ & \\
\hline Chemotherapy agents & & & 0.080 & & 0.773 \\
\hline 5-FU + cisplatin & $113(84)$ & $36(32)$ & & $10(9)$ & \\
\hline 5-FU + taxane & $15(11)$ & $1(7)$ & & $0(0)$ & \\
\hline 5-FU & $5(4)$ & $0(0)$ & & $0(0)$ & \\
\hline Cisplatin & $2(1)$ & $0(0)$ & & $0(0)$ & \\
\hline Dose (median/range) & 45 Gy (36 - 50.4 Gy) & & 0.357 & & 0.341 \\
\hline$\leq 41 \mathrm{~Gy}$ & $20(15)$ & $5(25)$ & 1.0 & $2(10)$ & 0.643 \\
\hline 45-50.4Gy & $115(85)$ & $32(28)$ & & $8(7)$ & \\
\hline Surgery & & & 0.322 & & 1.000 \\
\hline Thoraco-abdominal & $130(96)$ & $37(29)$ & & $10(8)$ & \\
\hline Transhiatal & $5(4)$ & $0(0)$ & & $0(0)$ & \\
\hline Resection status & & & 0.623 & & 0.126 \\
\hline Complete & $116(86)$ & $30(26)$ & & $7(6)$ & \\
\hline Microscopically involved margins & $12(9)$ & $5(42)$ & & $2(17)$ & \\
\hline Macroscopic residual tumor & $3(2)$ & $1(33)$ & & $1(33)$ & \\
\hline Unknown & $4(3)$ & $1(25)$ & & $0(0)$ & \\
\hline Planning & & & 0.125 & & 0.671 \\
\hline 3D & $116(86)$ & $31(27)$ & & $8(7)$ & \\
\hline IMRT & $2(1)$ & $2(100)$ & & $0(0)$ & \\
\hline VMAT & $17(13)$ & $4(23)$ & & $2(12)$ & \\
\hline POCO\# & & & & & 0.004 \\
\hline Yes & $37(27)$ & & & $7(19)$ & \\
\hline No & $98(73)$ & & & $3(3)$ & \\
\hline POPCO & & & $<0.001$ & & 0.247 \\
\hline Yes & $32(24)$ & $32(100)$ & & $4(13)$ & \\
\hline No & $103(76)$ & $5(5)$ & & $6(6)$ & \\
\hline Acute toxicity ${ }^{* * * *}$ & & & 0.457 & & 0.368 \\
\hline Yes & $15(11)$ & $6(40)$ & & $2(13)$ & \\
\hline No & $103(76)$ & $26(25)$ & & $8(8)$ & \\
\hline Unknown & 17 (13) & $5(30)$ & & $0(0)$ & \\
\hline
\end{tabular}

¥Some patients had one or more of the following: hypertension, chronic obstructive pulmonary disease, diabetes, heart attack in the year before surgery, liver cirrhosis (maximum Child-Pugh-Score=1).

*Patients with unknown grading have been excluded from analysis

**Patients with unknown ECOG-PS have been excluded from analysis

***from initial weight

$* * * *$ grade $\geq 3$ after N-RCT

\#POCO: Postoperative complications

¥POPCO: Postoperative pulmonary complications

\pm POD: Postoperative death

IMRT: Intensity Modulated Radiotherapie.

VMAT: Volumetric Modulated Arc Therapy

\section{Statistical analysis}

Fisher's exact test was used to evaluate the relationship between categorical clinical factors and the incidence of postoperative death or complications. Binary logistic regression analysis was used to analyze the relationship between incidence of postoperative death or complications and continuous clinical factors or dosimetric parameters derived from the lung DVH. In order to approximate and display linear dependencies between lung volume or dose and the interesting binary outcomes, predicted probabilities with $95 \%$ confidence bands were calculated based on linear regression analysis. Pearson correlation coefficient was used to quantify correlations between continuous dosimetric factors. All statistical tests were conducted two-sided at 0.05 level of significance.

\section{Results}

A total of ten postoperative deaths $(7 \%)$ were observed: 3 within 30 days (2\%) and 7 between 30 and
60 days after surgical intervention (5\%); no patient died during the operation. In the univariate analysis, weight loss ( $\geq 10 \%$ in 6 months prior to diagnosis, risk ratio: $1.60, \quad 95 \% \mathrm{CI}: \quad 0.856-2.992, \quad \mathrm{p}=0.043)$, ECOG-performance status (ECOG 2 vs. 1, risk ratio: 1.931, 95\% CI: 0.898-4.150, $\mathrm{p}=0.018$ ) and postoperative pulmonary plus non-pulmonary complications (risk ratio: 2.533 , 95\%CI: 0.978-6.563, $\mathrm{p}=0.004$ ) were significantly associated with postoperative mortality. There was no significant association between postoperative pulmonary complications and postoperative death $(p=0.247)$. There was no statistically significant correlation between irradiated lung volumes and postoperative pulmonary complications (Fig 1, Fig 2, Table 3). Lung V45 was the only variable which was significantly associated with higher incidence of postoperative pulmonary plus non-pulmonary complications $(\operatorname{Exp}(B): 1.285,95 \% C I$ 1.029-1.606, $\mathrm{p}=0.027)$, but not with the postoperative pulmonary complications $(\operatorname{Exp}(B): 1.249,95 \% C I$ 0.999-1.561, $\mathrm{p}=0.051)$. The median age of patients who died within sixty days after 
surgical resection was 5 years higher than that of other patients ( 66 vs. 61 years, $p=0.131$ ). There was no significant association between postoperative mortality and histology, operation technique, radiosensitizing agent or irradiated lung volumes. There was no significant difference between postoperative mortality in patients who were treated with a median radiation

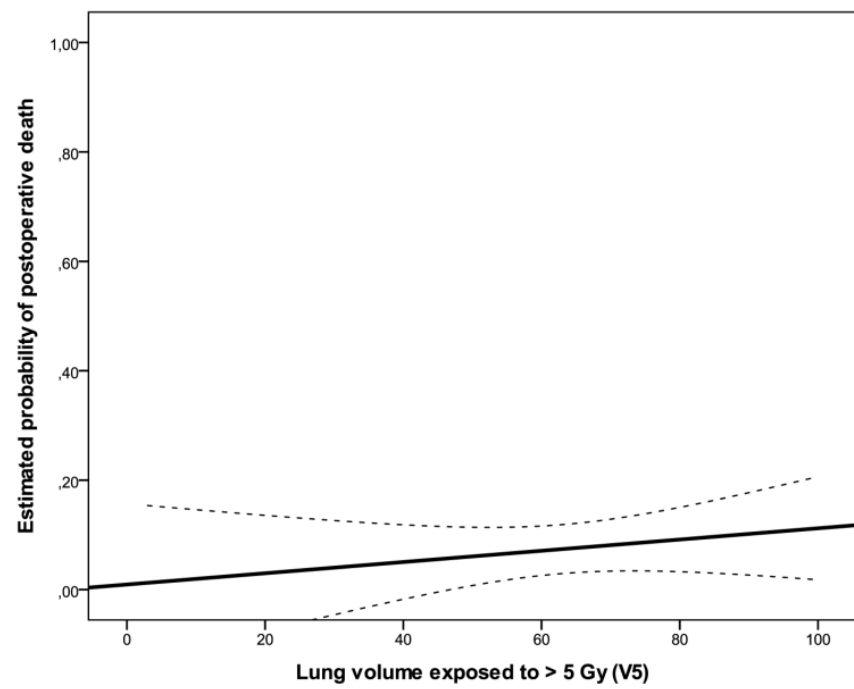

Figure 1. Estimated probability of death for lung V5 represented as solid line with $95 \%$ confidence bands (dashed lines). dose of 41 Gy (range, 36 - 41 Gy) and patients who were treated with a median dose of $45 \mathrm{~Gy}$ (range, 45 $50.4 \mathrm{~Gy}),(10 \%$ vs. $7 \%$, risk ratio: $0.720,95 \% \mathrm{CI}$ : $0.194-2.672, \mathrm{p}=0.643)$. There was no significant correlation between the incidence of acute toxicity $\geq$ grade 3 after $\mathrm{N}-\mathrm{RCT}$ and postoperative death $(\mathrm{p}=0.368)$.

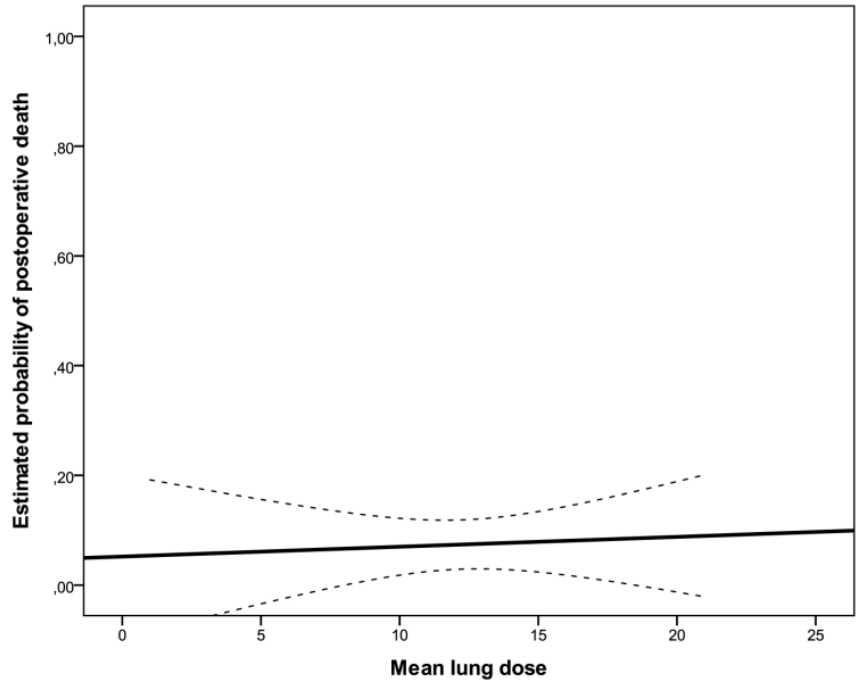

Figure 2. Estimated probability of death for mean lung dose represented as solid line with $95 \%$ confidence bands (dashed lines).

Table 3: Parameters calculated from the lung DVH and association with incidence of all serious postoperative complications, postoperative pulmonary complications and postoperative death

\begin{tabular}{|c|c|c|c|c|}
\hline Dose (median/range) & In $\% / \mathrm{cm}^{3} /$ Gy (range) & $\begin{array}{l}\text { POCO }^{*} \\
\operatorname{Exp}(B)(95 \% C I), p \text {-value }\end{array}$ & $\begin{array}{l}\text { POPCO ** } \\
\operatorname{Exp}(B)(95 \% C I), p \text {-value }\end{array}$ & $\begin{array}{l}\text { POD } \pm \\
\operatorname{Exp}(B)(95 \% C I), p \text {-value }\end{array}$ \\
\hline \multicolumn{5}{|l|}{ Lung (cumulative) } \\
\hline Mean dose & 13Gy (1-21Gy) & 1.056 (0.954-1.168), n.s. & 1.060 (0.952-1.179), n.s. & 1.027 (0.867-1.217), n.s. \\
\hline V5 & $65 \%(3-100 \%)$ & 1.004 (0.985-1.023), n.s. & 0.997 (0.977-1.017), n.s. & 1.017 (0.981-1.053), n.s. \\
\hline V10 & $50 \%(1-96 \%)$ & 1.009 (0.987-1.031), n.s. & 1.001 (0.979-1.024), n.s. & 1.008 (0.971-1.046), n.s. \\
\hline V15 & $36 \%(0-68 \%)$ & 1.016 (0.991-1.041), n.s. & 1.006 (0.981-1.032), n.s. & 1.008 (0.967-1.050), n.s. \\
\hline V20 & $17 \%(0-65 \%)$ & 1.027 (0.999-1.055), n.s. & 1.024 (0.995-1.053), n.s. & 1.000 (0.954-1.049), n.s. \\
\hline V30 & $8 \%(0-24 \%)$ & 1.045 (0.963-1.133), n.s. & 1.049 (0.964-1.142), n.s. & 0.985 (0.852-1.139), n.s. \\
\hline V40 & $3 \%(0-16 \%)$ & 1.140 (0.989-1.313), n.s. & 1.136 (0.983-1.312), n.s. & 0.864 (0.634-1.177), n.s. \\
\hline V45 & $1 \%(0-10 \%)$ & $\begin{array}{l}1.285(1.029-1.606) \\
p=0.027\end{array}$ & 1.249 (0.999-1.561), n.s. & 0.914 (0.603-1.385), n.s. \\
\hline V50 & $0 \%(0-4 \%)$ & 1.164 (0.571-2.377), n.s. & $1.263(0.618-2.581)$, n.s. & $0.000(0.000-0.000)$, n.s. \\
\hline \multicolumn{5}{|l|}{ PTV } \\
\hline Volume (cm3) & $\begin{array}{l}548 \mathrm{~cm}^{3}(120-2288 \\
\left.\mathrm{cm}^{3}\right)\end{array}$ & 1.000 (0.999-1.001), n.s. & 1.000 (0.999-1.001), n.s. & 1.001 (0.999-1.002), n.s. \\
\hline Vmean & 45Gy (28-52Gy) & 1.092 (0.956-1.247), n.s. & 1.078 (0.940-1.237), n.s. & 0.953 (0.792-1.146), n.s. \\
\hline
\end{tabular}

n.s. not significant

* POCO: Postoperative complications were defined as pneumonia, acute respiratory distress syndrome (ARDS), heart attack, sepsis and anastomosis insufficiency, which developed postoperatively within 60 days after surgery or before discharge.

** POPCO: Postoperative pulmonary complications were defined as ARDS and pneumonia, which developed postoperatively within 60 days after surgery or before discharge.

$\pm:$ POD: Postoperative death

\section{Discussion}

Although older individual studies have shown an increased risk of postoperative complications, the 4 most recent randomized trials published after the year
2000 have not shown an increase in postoperative complications and mortality rates in patients treated with surgery after N-RCT compared to patients treated with surgery alone (15). As demonstrated in the CROSS study (1) and retrospective studies (16) 
including the present one, TMT is associated with low rates of death within 30 days from surgery. In order to improve statistical power of our analysis, we focused on endpoints during an extended observation period of 60 days. Serious postoperative complications were observed in $27 \%$ of the patients, and their occurrence significantly increased the risk of postoperative death, as might be expected intuitively. In the study by Wang et al. (17), which included 444 patients, the most frequent complications were pulmonary (25\%) and gastrointestinal (23\%). Mean lung dose was strongly associated with pulmonary complications. An older and smaller study from the same institution was limited to 110 patients (18), but the results also suggested that pulmonary complications like pneumonia and ARDS correlated with several dosimetric parameters. In contrast, the study by Hurmuzlu et al. (19) did not find significant correlations between DVH parameters and postoperative pulmonary complications, nor increasing complications after TMT as compared to surgery alone. Hsu et al. (20) also reported lack of association between DVH parameters and pulmonary complications. However, preoperative forced expiratory volume 1 (FEV1) predicted development of complications. Unfortunately, lung function data were not available for our study. The number of patients was not sufficient for detailed analyses of individual comorbidities such as chronic obstructive pulmonary disease. Presence of any comorbidity or smoking status, a potential surrogate of worse pulmonary function, were not associated with any of the study endpoints (Table 2). It should also be noted that patients with impaired organ function typically are not offered TMT, which requires careful multi-disciplinary baseline assessment and discussion.

We observed correlations between complication rates and dosimetric parameters related to lung volume exposed to high radiation doses, especially V45. However, the primary study endpoint, mortality, was not influenced by these or other treatment-related variables. Only reduced ECOG performance status and greater weight loss significantly predicted higher rates of mortality. These factors might guide decision-making before proceeding to the surgical part of TMT. When interpreting our results, the limitations of this study must be acknowledged. These include risk of selection bias, as typical for retrospective studies, and limited statistical power of subgroup analyses despite the fact that a multicentric approach was chosen. One should also note that different centers used different treatment planning systems and dose calculation algorithms, which might have resulted in slightly different lung doses. With the inclusion criteria mentioned earlier, patients who might have de- veloped N-RCT-related toxicity that precluded them from surgical management were not entered into the database.

Regarding TMT in general, a vicious cycle exists: the larger the tumor and extent of disease involvement, the larger the radiation target volume. The larger the radiation target volume, the larger the irradiated lung volume (especially V5 and V10) (21). Extent of surgery with esophagectomy and lymphadenectomy also depends on disease involvement (duration of anesthesia, blood loss etc.). It is difficult to dissect the exact contribution of these factors and their complex interplay with comorbidities and age. Despite these limitations and caveats there is no reason to believe that higher lung doses provide any advantages. Therefore, currently recommended lung dose constrains should be respected to avoid lung toxicity (22-28).

\section{Conclusions}

Irradiated lung volumes did not show relevant association with intra- and postoperative mortality of patients treated with moderate median dose of $45 \mathrm{~Gy}$ of conformal normofractionated radiotherapy combined with standard radiosensitizing chemotherapy. Postoperative mortality was significantly associated with weight loss, poor performance status or incidence of postoperative complications in our study. Although statistically not significant, the incidence of postoperative mortality was lower in young patients. Decreasing the volume of lung receiving higher radiation doses might reduce the risk of postoperative complications.

\section{Competing Interests} interest.

The author(s) indicated no potential conflicts of

\section{References}

1. van Hagen P, Hulshof MC, van Lanschot JJ, et al. Preoperative chemoradiotherapy for esophageal or junctional cancer. N Engl J Med. 2012;366:2074-84.

2. Fakhrian K, Ordu AD, Lordick F, et al. Long-term outcomes of trimodality treatment of squamous cell carcinoma of esophagus with cisplatin and/or 5-FU. More than twenty years experience at a single institution. Strahlenther Onkol. 2014; [Epub ahead of print].

3. Fakhrian K, Ordu AD, Nieder C, et al. Outcomes of patients with squamous cell carcinoma of esophagus who did not receive the surgical part of tri-modality treatment. Anticancer Res; In press.

4. Fakhrian K, Ordu AD, Haller B, et al., Cisplatin- vs. oxaliplatin-based radiosensitizing chemotherapy for squamous cell carcinoma of the esophagus : A comparison of two preoperative radiochemotherapy regimens. Strahlenther Onkol. 2014;190:987-992.

5. Bauer K, Porzsolt F, Henne-Bruns D. Validity of the MAGIC study: sufficient for recommendations? Hepatogastroenterology. 2013;60:1822-4.

6. Siewert JR, Stein HJ, Feith M. Adenocarcinoma of the esophago-gastric junction. Scand J Surg. 2006;95:260-9.

7. Wang SL, Liao Z, Vaporciyan AA, et al. Investigation of clinical and dosimetric factors associated with postoperative pulmonary complications in esophageal cancer patients treated with concurrent chemoradiotherapy followed by surgery. Int J Radiat Oncol Biol Phys. 2006;64:692-9.

8. Nygaard K, Smith-Erichsen N, Hatlevoll R, et al. Pulmonary complications after bleomycin, irradiation and surgery for esophageal cancer. Cancer. 1978;41:17-22. 
9. Samel S, Hofheinz R, Hundt A, et al. Neoadjuvant radiochemotherapy of adenocarcinoma of the esophagogastric junction. Onkologie. 2001;24:278-282.

10. Whooley BP, Law S, Murthy SC, et al. Analysis of reduced death and complication rates after esophageal resection. Ann Surg. 2001;233:388-344.

11. Rodrigues G, Lock M, D'Souza D, et al. Prediction of radiation pneumonitis by dose-volume histogram parameters in lung cancer- a systematic review. Radiother Oncol. 2004;71:127-138.

12. Lee HK, Vaporciyan AA, Cox JD et al. Postoperative pulmonary complications after preoperative chemoradiation for esophageal carcinoma: correlation with pulmonary dose-volume histogram parameters. Int J Radiation Oncology Biol Phys. 2003;57:1317-1322.

13. Bosset JF, Gignoux M, Triboulet JP, et al. Chemoradiotherapy followed by surgery compared with surgery alone in squamous-cell cancer of the esophagus. N Engl J Med. 1997;337:161-7.

14. Fiorica F, Di Bona D, Schepis F, et al. Preoperative chemoradiotherapy for oesophageal cancer: a systematic review and meta-analysis. Gut. 2004;53:925-30.

15. Wilke TJ, Bhirud AR, Lin C. et al. A Review of the Impact of Preoperative Chemoradiotherapy on Outcome and Postoperative Complications in Esophageal Cancer Patients. Am J Clin Oncol. 2013; [Epub ahead of print].

16. Dähn D, Martell J, Vorwerk H, et al. Influence of irradiated lung volumes on perioperative morbidity and mortality in patients after neoadjuvant radiochemotherapy for esophageal cancer. Int J Radiat Oncol Biol Phys. 2010;77:44-52.

17. Wang J, Wei C, Tucker SL, et al. Predictors of postoperative complications after trimodality therapy for esophageal cancer. Int J Radiat Oncol Biol Phys. 2013;86:885-91.

18. Tucker SL, Liu HH, Wang S, et al. Dose-volume modeling of the risk of postoperative pulmonary complications among esophageal cancer patients treated with concurrent chemoradiotherapy followed by surgery. Int J Radiat Oncol Biol Phys. 2006;66:754-61.

19. Hurmuzlu M, Øvrebø K, Wentzel-Larsen T, et al. High-dose preoperative chemoradiotherapy in esophageal cancer patients does not increase postoperative pulmonary complications: correlation with dose-volume histogram parameters. Radiother Oncol. 2010;97: 60-4.

20. Hsu FM, Lee YC, Lee JM, et al. Association of clinical and dosimetric factors with postoperative pulmonary complications in esophageal cancer patients receiving intensity-modulated radiation therapy and concurrent chemotherapy followed by thoracic esophagectomy. Ann Surg Oncol. 2009;16:1669-77.

21. Fakhrian K, Oechsner M, Kampfer S, et al. Advanced techniques in neoadjuvant radiotherapy allow dose escalation without increased dose to the organs at risk: Planning study in esophageal carcinoma. Strahlenther Onkol. 2013;189:293-300.

22. Marks LB, Bentzen SM, Deasy JO, et al. Radiation dose-volume effects in the lung. Int J Radiat Oncol Biol Phys. 2010;76:70-6.

23. Czito BG, DeNittis AS, Palta M, et al. Chapter 53: Esophageal Cancer. In: Halperin EC, Wazer DE, Perez CA, Brady LW, ed. Perez and Brady's Principles and Practice of Radiation Oncology, $6^{\text {th }}$ ed. Philadelphia: Lippincott Williams \& Wilkins; 2013:1009-10

24. Emami B, Lyman J, Brown A, et al. Tolerance of normal tissue to therapeutic irradiation. Int J Radiat Oncol Biol Phys. 1991;21:109-122.

25. Jackson A, Marks LB, Bentzen SM, et al. The lessons of QUANTEC: Recommendations for reporting and gathering data on dose-volume dependencies of treatment outcome. Int J Radiat Oncol Biol Phys. 2010;76:155-160.

26. Marks LB, Bentzen SM, Deasy JO, et al. Radiation dose-volume effects in the lung. Int J Radiat Oncol Biol Phys. 2010;76:70-76.

27. Hernando ML, Marks LB, Bentel GC, et al. Radiation-induced pulmonary toxicity: A dose-volume histogram analysis in 201 patients with lung cancer. Int J Radiat Oncol Biol Phys. 2001;51:650-659.

28. Matzinger O, Gerber E, Bernstein Z, et al. EORTC-ROG expert opinion: radiotherapy volume and treatment guidelines for neoadjuvant radiation of adenocarcinomas of the gastroesophageal junction and the stomach. Radiother Oncol. 2009;92:164-175. 